\title{
Effects of Smartphone Sensor Variability in Road Roughness Evaluation
}

\begin{abstract}
Accelerometers embedded in smartphones have become an alternative means of measuring the roughness of roads. However, the differences in their sensitivity and sampling rates between smartphones could produce measurement inconsistencies that challenges the wide spread of the smartphone approach for road roughness measurements. In this study, the roughness measurement inconsistency was investigated between smartphones from three different brands. Using the same vehicle, device mount method, traversal speed, and method of producing a roughness index, field experiments demonstrated that accelerometer sensitivities and maximum sample rates vary significantly among smartphones of the same brand as well as across brands. For each smartphone, to achieve a margin-of-error within a $95 \%$ of confidence, significant large amounts of traversals are needed. Specifically, 24 and 35 traversals for a paved and an unpaved road, respectively. A higher sampling rate produced more consistent measurements and the least margin-of-error but resulted in larger data sizes. In addition, the measurements from all smartphones were not very sensitive to the size of the feature extraction window, therefore, selecting the largest practical window size will minimize the data size without significant loss of accuracy. This study indicated that for practical application, calibration is necessary to achieve consistent roughness measurements between various different smartphones.
\end{abstract}

KEYWORDS

Roughness, Road Impact

Factor, smartphones, accelerometer, feature extraction, connected vehicles.

\section{Introduction}

Each year the U. S. department of transportation and the states allocate most of their budget toward infrastructure maintenance, preservation, and rehabilitation. There are more than 4.1 million roadway miles in the U.S., and about one-third of those are unpaved gravel or dirt roads (Statistics 2018). State departments of transportation (DOTs) have developed pavement management systems (PMS) to manage the efficiency of network performance and to propose economical solutions for deteriorating pavements. PMS is a tool that collects data on pavement roughness and other surface distresses to evaluate the ride quality. A rougher roadway causes ride discomfort, risky driving behaviors, travel delays, excessive fuel consumption, and higher green-house gas emissions. Studies showed that distressed road surfaces increase fuel consumption by about $4 \%$ to $5 \%$ (Klaubert 2001).

Transportation agencies widely use the international roughness index (IRI) to quantify ride quality, which is an indicator of pavement condition. IRI produces longitudinal elevation profiles showing variation in surface roughness by using special inertial profiler vehicles, which require skillful labor to operate and to evaluate the data. Some shortcomings associated with this procedure such as the fixed reference speed of 80 kilometers-per-hour required by the golden car model (Bridgelall 2013), a high cost of about $\$ 6.12$ per mile (McGhee 2004), and wavelength biases due to the fixed quarter-car model used to calculate IRI (Marcondes et al. 1991).

The use of smartphones has become a potential alternative approach to measure roadway roughness to reduce cost, increase monitoring frequency, and increase coverage networkwide (Forslöf and Jones 2015). Most smartphone based methods for roughness measurement use the built-in sensors of smartphones, including the global positioning system (GPS) receiver, three-dimensional accelerometer with gyroscope, and ground speed sensor (Bridgelall et al. 2016b). The statistical model for road roughness improves when combining data from multiple sensors rather than taking only the magnitude from an accelerometer, referenced to an average speed (Douangphachanh and Oneyama 2014).

Case studies found that a given smartphone will generate consistent data for multiple traversals of a road segment to be used for roughness measurement (Zeng et al. 2018). However, for scenarios using multiple vehicles with varying suspension characteristics, a self-calibration approach using a machine learning could help to calibrate the output (Laubis et al. 2016). Nevertheless, variations in sensitivity among different smartphones present a challenge. There is strong ( $\mathrm{Li}$ and Goldberg 2018) to poor (Thiandee et al. 2019) correlation of the acceleration with the IRI when using different smartphone models or brands and calibration is then needed (Yang et al. 2020). It is possible to use data-mining algorithms to help mitigate issues with hardware diversity when relying on only on the capabilities of smartphones (Silva et al. 2017). Several studies validated the repeatability of smartphone-based measurements and their similarity with the IRI (Taleqani et al. 2019), (Douangphachanh and Oneyama 2013), (Darawade et al. 2016). However, none of the studies examined the impact of sensor variability among smartphones under the same measurement conditions.

This study is based on a method previously developed to quantify the ride quality of either paved or unpaved roads by applying a Road Impact Factor (RIF) transform to the inertial and geospatial position data collected (Bridgelall 2013). The RIFtransform produces RIF-indices that characterize roughness within adjustable distance windows along the traversal path. The window size tradeoff is that narrow windows more precisely localize the roughness caused by anomalies such as potholes, cracks, and rutting. In contrast, wider windows provide a greater reduction in data size and processing requirements at the expense of lower precision (Tai et al. 2010). Shorter window lengths are more sensitive to GPS positioning error (Douangphachanh and Oneyama 2014). Previous studies demonstrated that for any window size, the mean value of RIF-indices is directly proportional to the IRI, at any given speed (Bridgelall et al. 2016b). 
Based on the RIF index, this study is to characterize the measurement variability among smartphone sensors as a function of the RIF-transform window size by comparing measurements of roughness from multiple traversals of the same vehicle at the same speed on both paved and unpaved roads. The major contribution of this study is a quantification of the number of traversals needed for consistent roughness characterization using different smartphone sensors at different sampling frequencies, for paved roads and unpaved roads.

The organization of the remainder of this paper is: Section II describes the smartphone brands, their respective data collection apps, the method of generating roughness indices, the experimental design, and the data processing required for subsequent analysis. Section III produces the results and examines the differences in smartphone sensitivities as a function of feature extraction window size, and the margin-oferror as a function of the number of measurements taken for a paved road and an unpaved road. Section IV concludes this study with an observation about the effectiveness of a simple method of calibration and offers a recommendation for selecting the feature extraction window size in practice.

\section{Methodology and Experiments}

\section{A. Methodology}

The smartphones used for the data collection were an iPhone ${ }^{\circledR}$ 8 (i8), an iPhone® 10 (iX), and a Google Pixel (GP). The iPhones® ran the PAVET data collection app (Lu et al. 2019) in the $\mathrm{iOS} \circledast$ operating system, and the Google Pixel (GP) ran the RIVET data collection app (Lu et al. 2019) in the Android operating system. The apps logged signals from the three-axis accelerometer, ground speed sensor, timer, and gyroscope as inputs for the RIF-transform.

The RIF transform integrates a product of the vertical acceleration signal samples $\mathrm{gz}[\mathrm{n}]$ and the longitudinal velocity samples $v[n]$ as follows (Bridgelall et al. 2016b), (Taleqani et al. 2019):

$$
\mathrm{R}_{\Delta \mathrm{L}[\mathrm{w}]}=\sqrt{\frac{1}{\Delta \mathrm{L}[\mathrm{w}]} \sum_{\mathrm{n}=0}^{\mathrm{N}_{\mathrm{w}}-1}\left(\mathrm{~g}_{\mathrm{z}}[\mathrm{n}] \mathrm{v}[\mathrm{n}]\right)^{2} \Delta \mathrm{t}[\mathrm{n}]}
$$

where the RIF-index for distance window $w$ along the traversal path is $R_{\Delta L[w]}$ and $\Delta L$ is the window size. Within each window, $n$ is the sample instant. The index of the first and last samples of each window is 0 and $N_{w}-1$, respectively. The variable $N_{w}$ is the number of samples in window $w$. Since the smartphones do not sample the signals at a uniform rate, $\Delta T$ varies with each sample. The RIF-index is interpreted as the average g-force magnitude experienced per unit of distance $L$ traveled. The segment RIF-index $R_{\Delta L}$ is the mean of the RIF-indices across all windows of the entire segment such that:

$$
\mathrm{R}_{\Delta \mathrm{L}}=\frac{1}{\mathrm{~N}_{\mathrm{w}}} \sum_{\mathrm{w}=1}^{\mathrm{N}_{\mathrm{w}}} \mathrm{R}_{\Delta \mathrm{L}[\mathrm{w}]} .
$$

\section{B. Experimental Design}

Researchers can use a variety of vehicles to assess road quality. For example, some have used a motorcycle (Tai et al. 2010), (Setiawan and Nurdin), (Gorges et al. 2019) and others used a bicycle to assess the roughness of unmotorable roads as pedestrian paths and bicycle lanes (Zang et al. 2018), (Ho et al.
2016). This study used a regular passenger vehicle, a 2015 Volkswagen Jetta, to collect the data as illustrated in Fig.1 (a). An HTC smartphone was included with the other three smartphones described. However, it failed to capture GPS data and was excluded from the analysis. The phones were taped together onto the car floor behind the passenger seat as shown in Fig. 1 (b). The authors previously calibrated data from the i8 smartphone with data from a traditional road profiler to determine the proportionality constant with the IRI (Bridgelall et al. 2016a, Yang et al. 2020). Others demonstrated a similar utility by using a simple linear regression to calibrate smartphone accelerometer data with the IRI (Buttlar and Islam 2014). The two road segments, were tested in Fargo, North Dakota, including a 400-meter section of the paved road on 9th St NE, and a 300-meter section of the unpaved road on 57th St N, with portions as shown in Fig.1 (c, d).

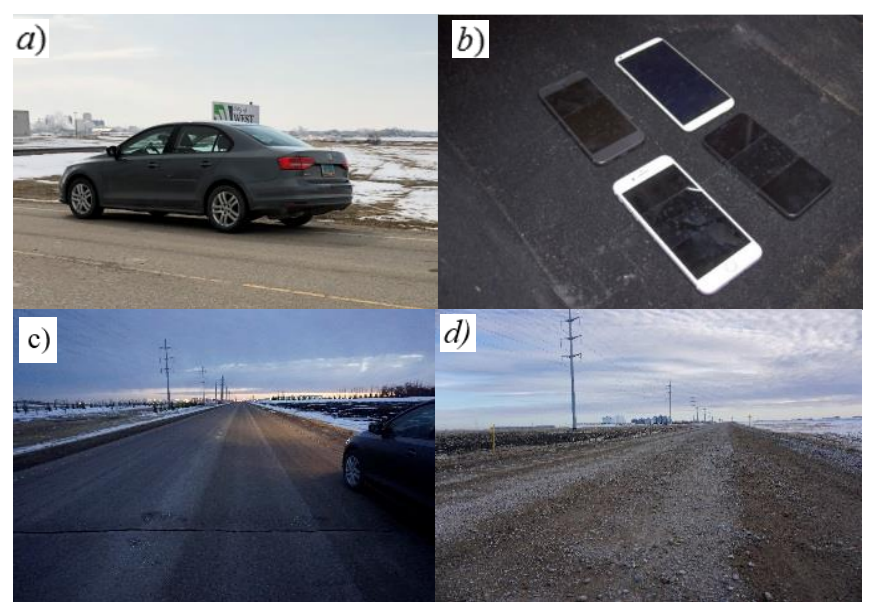

Figure 1. (a) 2015 Volkswagen Jetta used to collect data in this study, (b) Mounted smartphones, (c) Section of paved road, and (d) Section of unpaved road.

\section{Data Collection}

The authors used the same car to collect data by traversing the same segment of the paved and unpaved roads at approximately the same speed between $11.5 \sim 11.7 \mathrm{~m} / \mathrm{s}, 35$ times each. The smartphone apps transmitted the sampled data to a secure web server, which stored the data in comma-separated-value (CSV) format for post-analysis. The system stored the time instants in milliseconds, ground speed in meters-per-second, and the gyroscope angles of pitch, roll, and yaw in degrees. The sample rate of each phone varied substantially. The average sample rate of the Android and $\mathrm{iOS} \circledast$ smartphones was approximately 385 $\mathrm{Hz}$ and $85 \mathrm{~Hz}$, respectively. Similar studies used various smartphones with sampling frequencies ranging from $310 \mathrm{~Hz}$ to $10 \mathrm{~Hz}$ (Sattar et al. 2018). Table 1 summarizes the segment length traversed in meters $(\mathrm{m})$, the average traversal speed, and the average sample rate of each smartphone.

\begin{tabular}{ccccc} 
Table 1. Summary statistics for field experiment \\
\hline $\begin{array}{c}\text { Road } \\
\text { Type }\end{array}$ & $\begin{array}{c}\text { Phone } \\
\text { Type }\end{array}$ & $\begin{array}{c}\text { Segment } \\
\text { Length }(\mathrm{m})\end{array}$ & $\begin{array}{c}\text { Average } \\
\text { Speed }(\mathrm{m} / \mathrm{s})\end{array}$ & $\begin{array}{c}\text { Sample } \\
\text { Rate }(\mathrm{Hz})\end{array}$ \\
\hline \multirow{3}{*}{ Paved } & i8 & 399.821 & 11.772 & 86.924 \\
& iX & 399.819 & 11.751 & 87.894 \\
& GP & 399.965 & 11.753 & 386.488 \\
\hline \multirow{4}{*}{ Unpaved } & i8 & 299.823 & 11.548 & 79.088 \\
& iX & 299.810 & 11.543 & 80.364 \\
& GP & 299.968 & 11.559 & 385.548 \\
\hline
\end{tabular}




\section{Data Processing}

The data processing divided the vertical accelerations by 9.81 $\mathrm{m} \cdot \mathrm{s}^{-2}$ for normalization to $\mathrm{g}$-force values. Due to processing load variations, the average sample rate varied among smartphones and among traversals. Therefore, the data preparation procedure applied a digital noise filter to normalize the signal content within the nominal response frequency band (Bridgelall et al. 2016a), and to remove any constant offset that could bias the RIF-indices.

The authors used a technique previously reported (Bridgelall et al. 2016a) to align the spatial positions of each signal by using a known ground truth. This experiment used a rail-grade crossing bump on each road type. Using the signal peak produced from traversing the bump, interpolating the distances outwards, and then truncating all traversals to approximately the same length produced aligned signals for further processing. The paved road segment has a road width of $8 \mathrm{~m}$. The railroad grade crossing for the paved road as the maximum bump as shown in Figure 2 (a) has a length of $3 \mathrm{~m}$ and a width of $10 \mathrm{~m}$. The total paved road segment for testing is $580 \mathrm{~m}$. From the beginning point of the paved road test to the crossing is $420 \mathrm{~m}$, and from the ending point of the paved road test to the crossing is $160 \mathrm{~m}$. To be comparable to the paved road testing, the selected unpaved road segment in this field testing as shown in Figure 2 (b) has a width of $6 \mathrm{~m}$. This railroad grade crossing for this unpaved road as the maximum bump, which has a length of $2.5 \mathrm{~m}$ and a width of $6.7 \mathrm{~m}$. The total length of the unpaved road testing is $620 \mathrm{~m}$. From the beginning point of the unpaved road test to the crossing is $280 \mathrm{~m}$, and from the ending point of the unpaved road test to the crossing is $340 \mathrm{~m}$.

The interpolation of distance ( $\mathrm{Dn}$ ) based on the rail-grade crossing bump used the measured speed $(\mathrm{Vn})$ and time (tn) samples from the smartphones as follows:

$$
D_{n}=D_{n-1}+V_{n} \cdot\left(t_{n}-t_{n-1}\right) \cdot \frac{1}{1000} .
$$

The division by 1000 in Equation (3) converted the millisecond time intervals to seconds. Finally, an application of the RIF-transform to the aligned and equal length signals produced RIF-indices for distance window sizes of $1 \mathrm{~m}, 5 \mathrm{~m}$, $10 \mathrm{~m}, 15 \mathrm{~m}, 20 \mathrm{~m}$, and $40 \mathrm{~m}$.

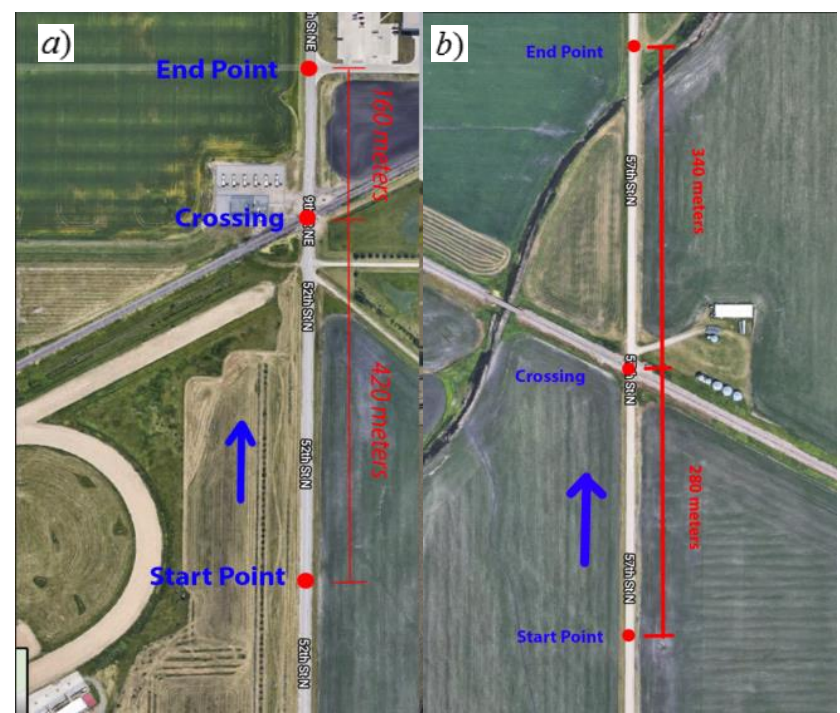

Figure 2. (a) Railroad crossing for the paved road (b) and unpaved road.

\section{Results and Discussions}

To characterize the measurement variability among smartphone sensors as a function of the RIF-transform window size and numbers of traversals for both paved and unpaved roads, the sensitivity difference and margin-of-error of the measured RIFindex from all the three smartphones are analyzed.

\section{A. Sensitivity Differences}

Fig. 3 plots the mean $(\mu)$ and standard deviation $(\sigma)$ of the RIFindices as a function of window size for paved (P) and unpaved (UP) roads. It is evident that there was general agreement among smartphone measurements that the unpaved road was more than twice as rough as the paved road. It is also evident that there were sensitivity differences among the smartphone sensors. For both road types, the i8 was consistently most sensitive and the Google Pixel was consistently least sensitive. This suggests that simple linear sensor calibration would produce consistent results.

For the unpaved road, there is a slight increase in the RIFindices with increasing window size, for all smartphones. The increase is proportionally more pronounced for the paved road. This increase is due to the end condition of the RIFtransformation where the last window is effectively shorter because it exceeds the signal stream. This results in a larger RIFindex per unit of distance. The effect is more pronounced for larger windows because there are fewer windows to dominate the effect of the last window of unequal size. The very low slope of the trend suggests that the mean of the RIF-indices is not very sensitive to the choice of window size.

The standard deviation of the RIF-indices for all smartphones and for both types of roads decreased with an increase in window size. This is intuitive because larger windows tend to average out roughness peaks and produce fewer RIF-indices, which consequently lowers the spread. This illustrates the tradeoff whereas larger windows reduce the spread in sensitivity among smartphones, narrower windows' increase the precision of localizing anomalies.

The Android smartphone exhibited the lowest standard deviation for each window size because its sampling frequency was more than 4.5 times greater than that of the iOS smartphones. Consequently, the low pass filter had a greater effect in reducing noise because the Nyquist frequency was substantially higher than the cut-off frequency. 


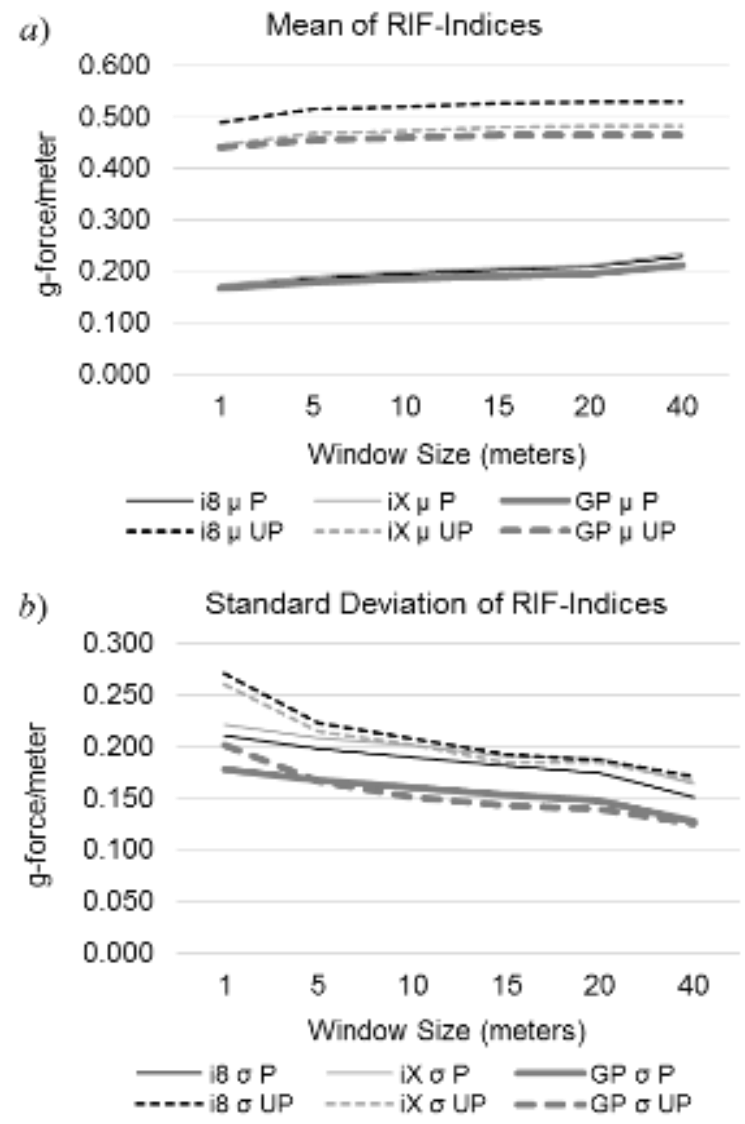

Figure 3. Mean (a) and standard deviation (b) of RIF-Indices.

\section{B. Margin-of-Error for RIF-Indices}

The margin of error (MOE) is a statistical measure that quantifies the spread in measurement variability within some confidence interval. Thus, a lower percentage provides high confidence in the observed values. The MOE percentage for the distribution of a random variable within a $(1-\alpha) \%$ confidence interval with significance $\alpha$ (Papoulis and Pillai 2002) is

$$
\mathrm{MOE}_{1-\alpha}= \pm \frac{\sigma t_{1-\alpha / 2, d f}}{\mu \sqrt{N}}
$$

where $t_{1-a / 2, d f}$ is the t-score for a normalized cumulative t-distribution with $d f$ degrees of freedom, $\mu$ is the mean value of the measurements, $\sigma$ is the standard deviation, and $N$ is the sample size.

Fig. 4 plots the $\mathrm{MOE}_{95}$ for the segment RIF-indices as a function of the traversal number. There are 35 traversals each for the paved and unpaved roads. It is clear that the $\mathrm{MOE}_{95}$ is not sensitive to window size because for each smartphone the trends are nearly identical and converges to approximately the same value. The number of traversals after which the $\mathrm{MOE}_{95}$ dropped below $2 \%$ consistently was different for each smartphone. For the paved road, approximately 24 traversals were needed to achieve lower than $2 \% \mathrm{MOE}_{95}$ for all smartphones and window sizes.
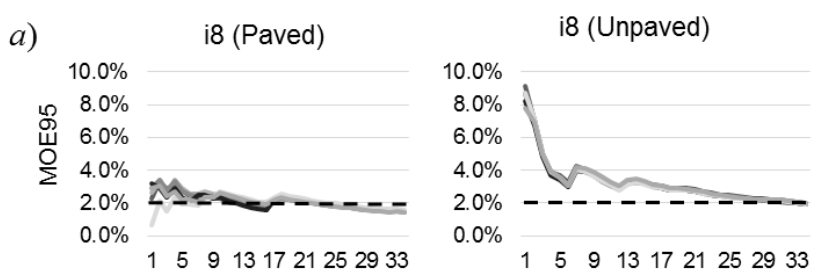

b)

iX (Paved)
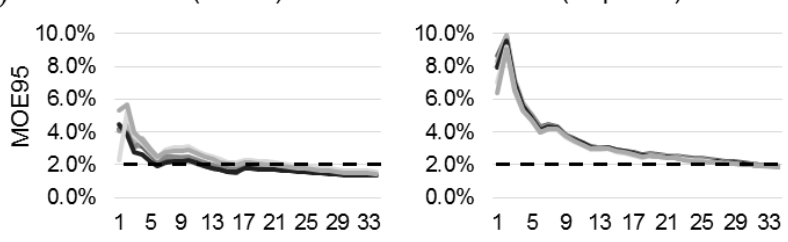

c)

$$
\text { GP (Paved) }
$$
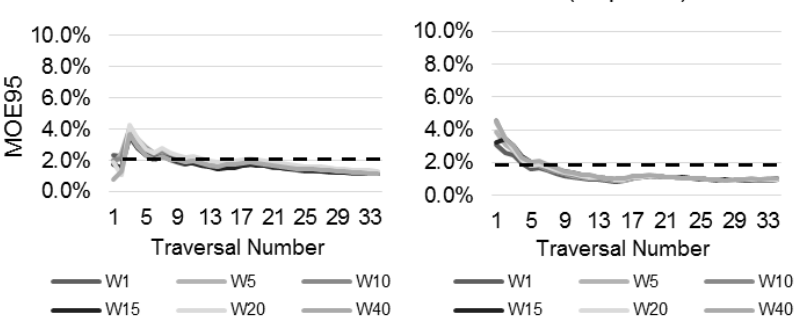

Figure 4. $\mathrm{MOE}_{95}$ as a function of traversals and window size.

The GP smartphone consistently produced lower than $2 \%$ MOE95 for the least number of traversals across all window sizes, and for both road types. This outcome was due to the higher sample rate that produced more consistent RIF-indices per traversal. As observed, the difference was more pronounced for unpaved roads. Roughness measurements for the unpaved road required approximately 35 traversals to achieve lower than $2 \%$ MOE95 for all smartphones and window sizes. This is an expected result because multiple traversals of an unpaved road will likely produce more variability in roughness than traversals of a paved road.

\section{CONCLUSIONS AND FUTURE WORK}

This study demonstrated that accelerometer sensitivities and maximum sample rates vary significantly among smartphones of the same brand as well as across brands. Therefore, when the smartphones are used to measure the ride quality of roads, these differences result in inconsistent measurements. However, the measurement trends with feature extraction window size and margin-of-error are nearly identical. This suggests that using a simple calibration constant would normalize the measurements for consistent results across phones that are mounted in a specific vehicle. Subsequently, the nominal ride quality experienced with different vehicles would be the statistical average of the roughness indices that each vehicle produced.

Roughness measurements are not very sensitive to window size selection, particularly for rougher rides across unpaved and gravel roads. For all smartphones and feature extraction window sizes, the MOE95 dropped below 2\% after 24 and 35 traversals for the paved and unpaved roads, respectively. A higher sample rate produces more consistent measurements but also results in a larger data size per traversal. Since measurements are not very sensitive to feature extraction window sizes, using larger 
windows could help to reduce the data size and improve the measurement consistency. However, the tradeoff is a loss of precision in the localization of roadway anomalies. Hence, the recommended approach is to use the largest feature extraction window size tolerable so that the localization of anomalies is within practical limits. Future work will examine the effectiveness of calibrating multiple smartphones in a single reference vehicle, and then using them in many vehicles with various suspension characteristics to estimate the consistency of the average ride quality experienced on a given roadway, at various speeds.

\section{References}

Bridgelall, R. 2013. Connected vehicle approach for pavement roughness evaluation. Journal of Infrastructure Systems, 20(1), 04013001.

Bridgelall, R., et al. 2016a. Precision enhancement of pavement roughness localization with connected vehicles. Measurement Science and Technology, 27(2), 025012.

Bridgelall, R., et al. 2016b. Use of connected vehicles to characterize ride quality. Transportation Research Record, 2589(1), 119-126.

Buttlar, W. G. and Islam, M. S., 2014. $0981 Y 04$ Integration of Smart-phone-based Pavement Roughness Data Collection Tool with Asset Management System. Purdue University-Discovery Park.

Darawade, K., et al. 2016. Estimation of road surface roughness condition from android smartphone sensors. Int. J. Recent Trends in Eng. \& Research, 2(3), 339-346.

Douangphachanh, V. and Oneyama, H., Estimation of road roughness condition from smartphones under realistic settings. ed. 2013 13th international conference on ITS telecommunications (ITST), 2013, 433-439.

Douangphachanh, V. and Oneyama, H., Exploring the use of smartphone accelerometer and gyroscope to study on the estimation of road surface roughness condition. ed. 2014 11th International Conference on Informatics in Control, Automation and Robotics (ICINCO), 2014, 783-787.

Forslöf, L. and Jones, H. 2015. Roadroid: Continuous road condition monitoring with smart phones. Journal of Civil Engineering and Architecture, 9(4), 485-496.

Gorges, C., Öztürk, K. and Liebich, R. 2019. Impact detection using a machine learning approach and experimental road roughness classification. Mechanical Systems and Signal Processing, 117, 738-756.

Ho, C.-H., Lai, C.-P. and Almonnieay, A., Using Geographic Information Systems and Smartphone-Based Vibration Data to Support Decision Making on Pavement Rehabilitation. ed. International Conference on Information Processing and Management of Uncertainty in Knowledge-Based Systems, 2016, 475485.

Klaubert, E. C., 2001. Highway effects on vehicle performance. United States. Federal Highway Administration.

Laubis, K., Simko, V. and Schuller, A. 2016. Road condition measurement and assessment: A crowd based sensing approach.

Li, X. and Goldberg, D. W. 2018. Toward a mobile crowdsensing system for road surface assessment. Computers, Environment and Urban Systems, 69, 51-62.

Lu, P., et al., 2019. Intelligent Transportation Systems Approach to Railroad Infrastructure Performance Evaluation: Track Surface Abnormality Identification with Smartphone-Based App.

Marcondes, J., et al. 1991. Spectral analysis of highway pavement roughness. Journal of Transportation engineering, 117(5), 540-549.

McGhee, K. H., 2004. Automated pavement distress collection techniques. Transportation Research Board.

Papoulis, A. and Pillai, S. U., 2002. Probability, random variables, and stochastic processes. Tata McGraw-Hill Education.

Sattar, S., Li, S. and Chapman, M. 2018. Road surface monitoring using smartphone sensors: A review. Sensors, 18(11), 3845.

Setiawan, E. B. and Nurdin, H. Road Quality Assessment Using International Roughness Index Method and Accelerometer on Android. Lontar Komputer: Jurnal Ilmiah Teknologi Informasi, 62-72.

Silva, N., et al. 2017. Anomaly detection in roads with a data mining approach. Procedia computer science, 121, 415422.

Statistics, A. A., 2018. National Transportation Safety Board.

Tai, Y.-c., Chan, C.-w. and Hsu, J. Y.-j., Automatic road anomaly detection using smart mobile device. ed. conference on technologies and applications of artificial intelligence, Hsinchu, Taiwan, 2010.

Taleqani, A. R., et al. 2019. Data Driven Analytics of Road Quality. Proceedings of 34th International Confer, 58, 454-463.

Thiandee, P., et al. 2019. An Experiment on Measurement of Pavement Roughness via Android-Based Smartphones. arXiv preprint arXiv:1907.13120.

Yang, X., et al. 2020. Calibration of Smartphone Sensors to Evaluate the Ride Quality of Paved and Unpaved Roads. International Journal of Pavement Engineering, 2020, https://doi.org/10.1080/10298436.2020.1809659.

Zang, K., et al. 2018. Assessing and mapping of road surface roughness based on GPS and accelerometer sensors on bicycle-mounted smartphones. Sensors, 18(3), 914.

Zeng, H., et al. 2018. Feasibility assessment of a SmartphoneBased application to estimate road roughness. KSCE Journal of Civil Engineering, 22(8), 3120-3129. 